\title{
Accidental ingestion of a dental bur in an 84 year-old-male
}

Francesk Mulita ${ }^{1}$, Georgios-Ioannis Verras ${ }^{1}$, Elias Liolis ${ }^{1}$, Levan Tchabashvili ${ }^{1}$, Ioannis Panagiotopoulos $^{1}$, Charalampos Kaplanis ${ }^{1}$, Ioannis Perdikaris ${ }^{1}$, and Konstantinos Bouchagier $^{1}$

${ }^{1}$ University General Hospital of Patras Holy Mary the Help

January 23,2022

\begin{abstract}
This report describes the case of an 84-year-old male who was brought to the emergency room because a dental bur was swallowed accidentally during a dental procedure. The foreign body was successfully removed by gastroenterologists endoscopically 8 days after the ingestion and was identified as a 2-cm-long dental bur.
\end{abstract}

CASE REPORT

\section{Accidental ingestion of a dental bur in an 84 year-old-male}

Francesk Mulita ${ }^{1}$, Georgios-Ioannis Verras $^{1}$, Elias Liolis ${ }^{2}$, Levan Tchabashvili ${ }^{1}$, Ioannis Panagiotopoulos $^{3}$, Charalampos Kaplanis ${ }^{1}$, Ioannis Perdikaris ${ }^{1}$, Konstantinos Bouchagier ${ }^{1}$

${ }^{1}$ Department of General Surgery, General University Hospital of Patras, Greece

${ }^{2}$ Department of Internal Medicine, General University Hospital of Patras, Greece

${ }^{3}$ Department of Cardiothoracic Surgery, General University Hospital of Patras, Greece

Correspondence: Francesk Mulita MD, MSc, $\mathrm{PhD}(\mathrm{c})$, Resident Surgeon at the Department of Surgery, General University Hospital of Patras, Achaia, Greece, Tel.: +30 6982785142; E-mail:oknarfmulita@hotmail.com; ORCID Id:https://orcid.org/0000-0001-7198-2628

\begin{abstract}
This report describes the case of an 84-year-old male who was brought to the emergency room because a dental bur was swallowed accidentally during a dental procedure. The foreign body was successfully removed by gastroenterologists endoscopically 8 days after the ingestion and was identified as a 2-cm-long dental bur.
\end{abstract}

Key words: Foreign body, endoscopy, x-rays

Key Clinical Message

Although the majority of ingested foreign bodies pass through the gastrointestinal tract, endoscopic intervention is necessary in $10-20 \%$ of these cases. 


\section{Introduction}

Ingestion of foreign bodies is very common. Especially, more than 120.000 cases were recorded in the USA in only one year, while approximately 1500 deaths per year are attributed to ingestion of foreign bodies [1-2]. Various complications can be occurred during daily clinical practice by accidental ingestion of dental objects such as burs, impression materials, dental inlays or crowns, endodontic posts, fixed or removable prosthetic restorations [3]. These life-threatening complications like peritonitis, sepsis, fistulas and duodenocolic fissures, abscess formation and injury to the digestive tract are related to obstruction or perforation caused by the ingested foreign body $[1,3]$. Ingested foreign bodies usually pass through the anus without any complication. In 10-20\% of patients endoscopic removal is required, while in $1 \%$ of patients surgery is necessary [3]. We herein report a case of an accidental ingestion of a dental bur in an 84 yearold-male.

\section{Case Report}

An 84-year-old male with atrial fibrillation, hypertension and type 2 diabetes mellitus was brought to the emergency room of our hospital by a private dental practitioner. The dentist disclosed that a 2 -cm dental bur was swallowed accidentally by his patient 2 hours ago during the dental procedure. On examination, the patient's temperature was 36.5, heart rate was 71 beats per minute, blood pressure was 151/87 and respiratory rate was 17 breaths per minute. His abdomen was soft, without distension and with no evidence of palpable mass. His routine blood tests including haemogram, C-reactive protein level, liver and renal function test, serum amylase and lipase were normal. Chest radiography showed no evidence of free air and no other abnormalities. X-ray of the abdomen revealed a hyperdense, foreign body in the left lower quadrant (Fig. 1A). Upper endoscopy was not performed as the foreign body was shown distal to stomach - not accessible to endoscopy.

Patient was kept under close observation in the department of surgery. He was kept under high fibrous diet and $20 \mathrm{ml}$ of oral gastrografin was prescribed every 8 hours for allowing the rapid transport of the foreign object within the gastrointestinal (GI) tract. Serial radiographic examinations were performed to monitor the migration of the ingested foreign body. A repeated x-ray of the abdomen was taken 24 hours after the ingestion of the object. This radiograph suggested the presence of the foreign body in the right lower quadrant of the abdomen (Fig. 1B). As the patient did not agree to make any endoscopic or surgical intervention, abdominal radiographies were performed 48 hours (Fig.1 C), 72 hours (Fig.1 D), 5 days (Fig.1 E) and 7 days (Fig.1 F) after the foreign body ingestion, but the object remained in the same position.

An attempt was made by gastroenterologists to remove the foreign body endoscopically. Eventually, anterograde colon preparation and colonoscopy was performed. The foreign body was detected in terminal ileum, was grasped with an endoscopic loop, pulled through the valve and removed per anus. The length of the dental bur was about $2 \mathrm{~cm}$. The patient did not have any complication and he was mobilized 2 hours after the foreign body removal. He was started on oral diet 12 hours afterwards and was discharged home in a very good condition 24 hours after the endoscopy. 


\section{Discussion}

Ingestion of foreign body is commonly encountered in radiological practice and its significance should not be underestimated [1]. Whenever a dentist loses a dental material or any other foreign object inside oral cavity, a radiologist must be consulted even if the patient has no symptoms [4]. Usually, the foreign bodies pass through the GI tract and are evacuated in 2 to 5 days without symptoms [3]. The two most common locations of an ingested foreign object at the initial presentation is in the stomach (58.1\%) and small intestine $(32.7 \%)$ [5]. Endodontic files have been reported to pass through GI tract within 3 days, while 10-20\% require endoscopic intervention and only in $1 \%$ surgery is necessary [6].

Radiographic examination with chest and abdominal x-ray is necessary for determining the location, size and nature of the ingested foreign object [7]. The computed tomography (CT) scan can also be used to localize foreign objects, but it is usually performed to define the exact extent of injury to the involved organs and the damage to the surrounding tissues [1]. In our case, the foreign body was radio opaque and the size assessment was noted. We did not perform a CT scan as the foreign body was identified in radiography and the patient had normal blood tests and no symptoms.

Size, sharpness and shape of the ingested foreign body are some characteristics that should be taken into consideration when a medical professional has to deal with this condition. The risk of injury increases when the size of the object is more than $5 \mathrm{~cm}$ or has a pointed shape [8].

According to the literature, the removal of a foreign object that has entered the gastrointestinal tract is determined on the basis of patients' age, size shape and location of the object, and time since the ingestion. The risk of complications such as obstruction or perforation determines the timing of endoscopy [9]. The overall rate of perforation caused by ingested foreign bodies is in the range of 1-7\%. However, the incidence is increased to $15-35 \%$, when pointed or sharp foreign bodies are considered [10]. According to Bondarde $\mathrm{P}$ et al., the management of sharp bodies such as endodontic file, when lodged in GI tract, is endoscopic retrieval or the careful monitoring with periodic radiographs. If the foreign body does not succeed to progress after 72 hours or complications such as perforation, obstruction or bleeding are noticed, surgery should be preferred immediately [4]. In our case, periodic abdominal radiographies were performed for 7 days, because the patient did not agree to make any endoscopic or surgical intervention the first 7 days. Oral gastrografin was prescribed for allowing the rapid transport of the foreign body within the GI tract. The barium sulfate was not preferred to be used because of its side-effects such as constipation and mechanical obstruction of bowel, which can make it difficult to perform endoscopy [11]. In addition, laxative treatment should be given to patients after barium studies to reduce the incidence of colonic retention of barium sulfate [12]. Fortunately, the foreign body which was identified as a 2-cm-long dental bur was successfully removed by gastroenterologists endoscopically 8 days after the ingestion.

\section{Conclusion}

Iatrogenic accidents during routine dental procedures are common and unpredictable. Early recognition and diagnosis of this condition is the key to prevent serious complications. In 10-20\% of these cases endoscopic intervention is necessary and less than $1 \%$ of patients require surgical retrieval. In our case the foreign body was removed by gastroenterologists endoscopically.

\section{Ethical approval and consent to participate}

A written informed consent was obtained from the patient for publication of this case report.

\section{Conflict of interest}

There are no conflicts of interest to declare. 


\section{Financial support and sponsorship}

None

\section{Author contribution}

FM, G-IV, EL, LT, IP, IP and CK: contributed to the clinical data collection and prepared the case report. FM and KB: contributed to the design of the case report presentation and performed the final revision of the manuscript.

\section{Data availability}

Data available on request from the authors

\section{Acknowledgments}

This paper was accepted and presented as an e-poster in the $32^{\text {th }}$ Hellenic Congress of Surgery 2021 and appears online in part.

\section{References}

1. Guelfguat M, DiPoce J, DiPoce J. A dental nightmare, resolved: what a radiologist needs to know when consulted about ingestion of dental foreign body material. BJR Case Rep 2016; 2: 20150166.

2. Amarlal D, Jeevarathan J, Muthu MS, Venkatachalapathy A, Rathna Prabhu V. Iatrogenic accidental ingestion of a dental bur. Indian J Pediatr . 2009;76(3):333-334. doi:10.1007/s12098-009-0003-7

3. Dionysopoulos D. Accidental ingestion and aspiration of foreign objects during dental practice. Stomatological Dis Sci2017;1:87-9.

4. Bondarde P, Naik A, Patil S, Shah PH. Accidental Ingestion and Uneventful Retrieval of an Endodontic File in a 4 Year Old Child: A Case Report. J Int Oral Health . 2015;7(Suppl 2):74-76.

5. Antoniou D, Christopoulos-Geroulanos G. Management of foreign body ingestion and food bolus impaction in children: a retrospective analysis of 675 cases. Turk J Pediatr 2011; 53: 381-7.

6. Bhatt R, Atrey A, Kaur A, Dave L. Accidental ingestion of a dental bur seen in a paediatric patient A case report. Adv Hum Biol 2013;3(3):82-4.

7. Daneswari V, Visalaxi, Harika R (2016) Emergency Management of an Accidental Ingestion of a Dental Foreign Body in Pediatric Patient Using Rigid Esophagoscopy - A Case Report. Pediatr Dent Care 1:111. doi:10.4172/pdc.1000111

8. Jaan A, Mulita F. Gastrointestinal foreign body . 2020 Sep 4. In: StatPearls [Internet]. Treasure Island, FL: StatPearls Publishing; 2020 Jan-. PMID: 32965874.

9. Mulita F, Papadopoulos G, Tsochatzis S, Kehagias I. Laparoscopic removal of an ingested fish bone from the head of the pancreas: case report and review of literature. Pan Afr Med J . 2020;36:123. Published 2020 Jun 25. doi:10.11604/pamj.2020.36.123.23948

10. Brinster CJ, Singhal S, Lee L, Marshall MB, Kaiser LR, Kucharczuk JC. Evolving options in the management of esophageal perforation. Ann Thorac Surg . 2004;77(4):1475-1483. doi:10.1016/j.athoracsur.2003.08.037

11. Khartade HK, Meshram VP, Tumram NK, Parchake MB, Pathak HM. Fatal Aspiration of Barium Sulfate in a Case of Myasthenia Gravis: A Case Report and Review of Literature. J Forensic Sci. 2020 Jul;65(4):1350-1353. doi: 10.1111/1556-4029.14297

12. Pathan S, Benzar T, Master S, Peddi P. Iatrogenic constipation from barium blockade: A case report. Clin Case Rep . 2019;7(8):1562-1564. Published 2019 Jul 10. doi:10.1002/ccr3.2280 
Figures
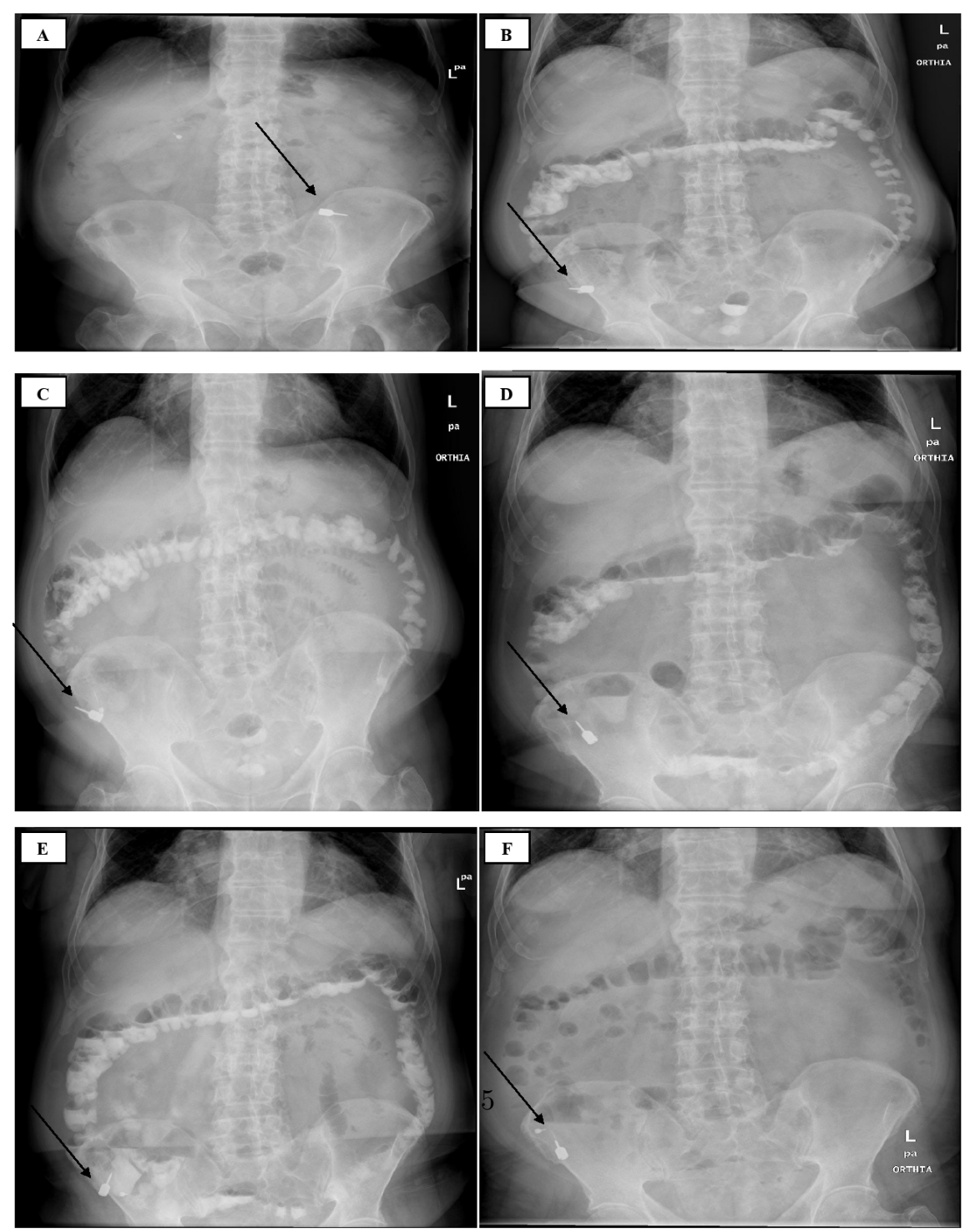
Fig. 1. Abdominal radiography: Showed a hyperdense, foreign body in the left lower quadrant 2 hours after ingestion (A). Movement of foreign body in the right lower quadrant 24 hours after ingestion (B). The foreign body remains in the same position 48 hours (C), 72 hours (D), 5 days (E) and 7 days (F) after ingestion.
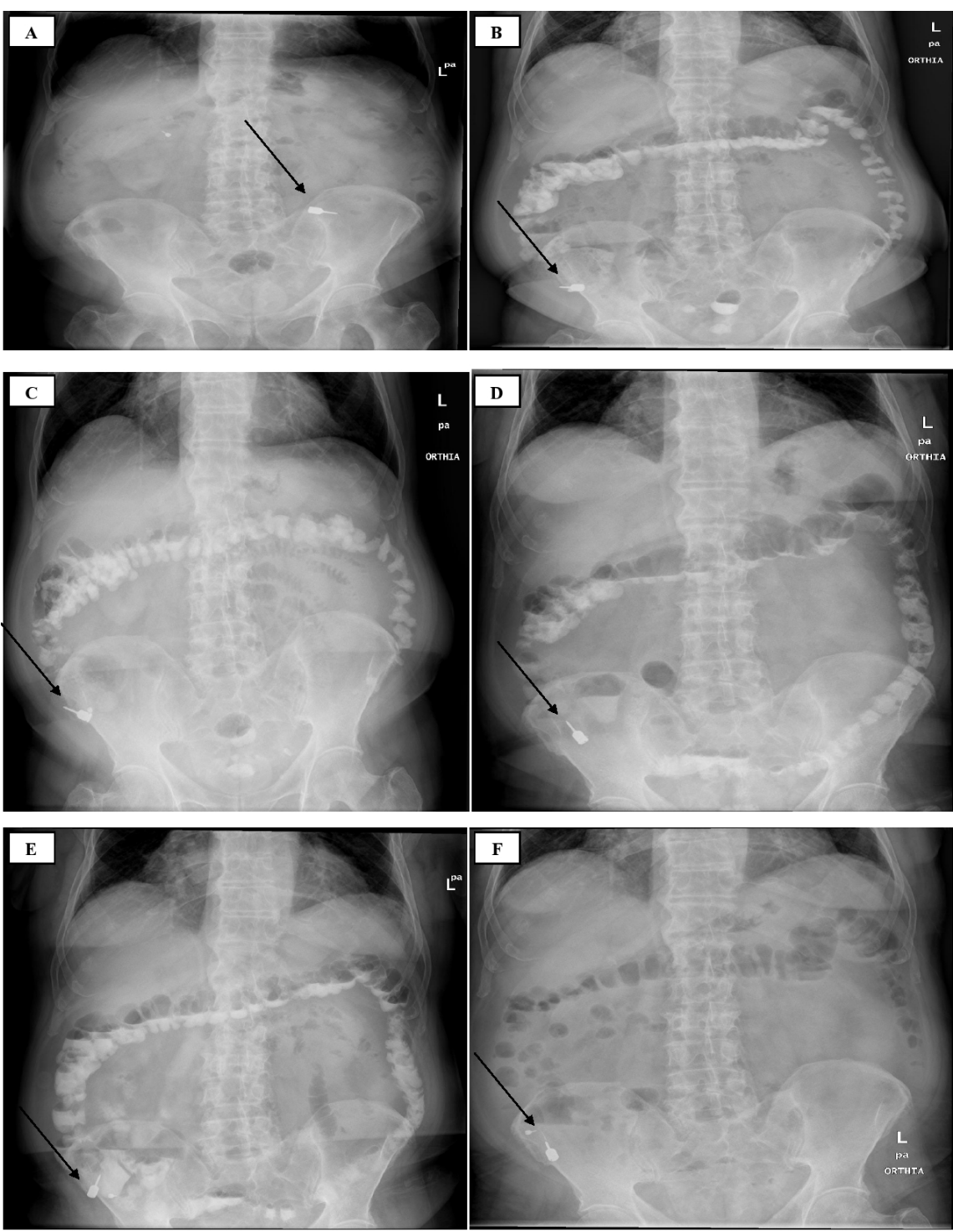